\title{
Estrategias de Afrontamiento de Padres de Niños con Secuelas de Quemaduras Visibles
}

\section{Coping Strategies Used by Parents of Children with Visible Burn sequels}

Evelyn Lagos H.*, Margarita Loubat O.**, Elizabeth Muñoz A.*, Paula Vera de la F.*

\begin{abstract}
Resumen
La presente investigación tuvo por objeto relacionar las estrategias de afrontamiento de padres de niños quemados con los niveles de ansiedad presentados por sus hijos. Para ello, se utilizó una metodología cuantitativa y un diseño de tipo descriptivo-correlacional.

La muestra estuvo conformada por 49 niños (as) y sus padres que los acompañaban en su tratamiento en COANIQUEM. Los instrumentos utilizados fueron el Inventario de Ansiedad Estado-rasgo para Niños de Spielberger y el Cuestionario de Modos de Afrontamiento de Lazarus y Folkman.

Los resultados señalan que las estrategias de afrontamiento Autocontrol, Reinterpretación positiva, Resolución planificada y Aceptación de la responsabilidad correlacionan significativamente con bajos niveles de ansiedad en los niños quemados.
\end{abstract}

Palabras claves: Niños quemados, Estrategias de afrontamiento y Ansiedad.

\begin{abstract}
This research was aimed at relating the coping strategies developed by parents of children suffering from burn injuries and the levels of anxiety presented by the children themselves. For this purpose, a quantitative methodology and a design of the descriptive correlacional type were used.

The sample consisted of 49 children, and their accompanying parents, attending treatment at COANIQUEM. The instruments used were Spielberger's Inventory of Anxiety State - feature for Children and the Lazarus and Folkman's Ways of Coping Questionnaire. The results indicate that the strategies coming of selfcontrol, Positive Reinterpretation, Planned Resolution and Acceptance of Responsibility correlate significantly with low levels of anxiety in burned children.
\end{abstract}

Key Words: Burned children, Coping Strategies and Anxiety.

* Psicólogas Universidad de Santiago de Chile.

** Psicóloga y Profesora Escuela de Psicología, Universidad de Santiago de Chile. E:mail mloubat@lauca.usach.cl. 


\section{Introducción}

Desde una perspectiva integral de salud se puede definir el accidente de quemadura en niños como un evento que los afecta física, psicológica y socialmente. En efecto, una situación estresante como ésta produce una serie de cambios en el aspecto físico que repercuten entre otros, en la autoimagen, autoestima y en la interrelación.

Sabemos, que producto de las quemaduras y sus consecuencias se genera en el menor miedo a la muerte, a la desfiguración y al abandono; naturalmente esto influye en la posible aparición de sintomatología ansiosa y depresiva (Ramírez, 2001) y desde luego en el proceso de adaptación del niño a la quemadura (Artz \& Moncrief, 1972).

Estas reacciones emocionales negativas, si bien están presentes durante toda la evolución de la lesión se intensifican en el período de resocialización posterior a la quemadura. Esto se produce -fundamentalmente- en el curso de los dos primeros años después de ocurrido el accidente e influyen en ello, la necesidad de usar prendas compresivas, el estrés emocional para adaptarse a los cambios físicos y psicológicos, en ciertos casos la cirugía reparadora y en fin, múltiples intervenciones que tienen que ver con el tratamiento y la rehabilitación en general. (Bendlin, Linares, Benaim, 1993).

La presencia y manifestación de estas reacciones negativas en el niño, se ven ciertamente influidas por la forma en que los padres se adapten a esta situación estresante (Capdeville \& Medina, 1998), esto "debido a que ellos son el principal armazón para las adaptaciones hechas por el niño" (Clapp, 1979; Ross, 1978; Tylke, 1980 citado en Ugalde, 1998). Consecuentemente a ello, Breton, Valla y Lambert (1993, citado en Hermosilla \& Montt, 2001) postulan, que la repuesta del niño al estresor se relaciona con la respuesta de los padres a éste.
Castillo y Santander (1996), se refieren a las reacciones de la familia y de los padres del niño cuando se enfrentan a una quemadura, señalando diversas etapas de ajuste. Estas etapas se caracterizan en un primer momento por la desorganización, confusión y angustia; posteriormente y en algunos casos por fuertes sentimientos de culpa, en otros por rabia e impotencia. Estos estados emocionales provocarían un bloqueo en las habilidades parentales de acogida y entrega de afecto, como también en aquellas de control y guía de sus hijos.

Ya superada la crisis inicial, las autoras citadas señalan que surgen conflictos relacionales entre los padres, también entre padres e hijos y con el niño quemado en particular, debido a una fuerte tendencia a la sobreprotección de éste. En otras ocasiones, se observan respuestas de negación y abandono hacia este niño "difícil" y "distinto" por parte de los padres, lo que se traduce en dificultades en el cumplimiento del tratamiento.

En la etapa final, según Castillo y Santander, los padres ejercitarían nuevas formas de relacionarse al interior de la familia e iniciarían una nueva organización, incorporando el tratamiento del niño, apoyando su reinserción social y avanzando hacia la aceptación de la secuela.

Estas reacciones emocionales manifestadas por los padres, recientemente señaladas, constituyen una de las principales fuentes de estrés infantil. "Se ha observado, que aunque los padres y familiares traten de ocultar estos sentimientos a través de un relato verbal que minimiza la situación relacionada con el suceso estresante, es la actitud emocional la que prevalece fundamentalmente e impresiona mas al menor". Esta influencia parental, generalmente se debe a que en los niños pequeños resulta de capital importancia la valoración que del suceso o la situación hagan los padres o criadores; una respuesta positiva de éstos puede amortiguar el impacto estresante sobre el niño; por el contrario, si 
el progenitor se muestra ansioso ante el acontecimiento, el niño fácilmente imitará la respuesta emocional del adulto, con lo que aumentará la probabilidad de que éste al enfrentarse de nuevo a una situación similar, muestre respuesta de afrontamiento ineficaces (Palomo del Blanco, 1995; Buendía \& Mira, 1993). Además, sabemos que en la vida de un niño, los padres funcionan como un filtro protector que elabora las experiencias haciéndoselas más tolerables al menor. Es por ello que la forma en que ambos padres afronten el accidente y posterior tratamiento, resulta fundamental en la ayuda que le puedan dar a su hijo a lo largo del mismo (Palanca \& Pedreira, 2002). Por lo tanto, frente a los accidentes de quemaduras no se puede separar la reacción de los padres y el afrontamiento de la familia, de la reacción del niño. Es por ello que cuando un niño se quema, la lesión que se provoca trasciende el daño tisular afectando biopsicosocialmente al menor y su familia (Capdeville \& Medina, 1998). Esta afirmación obliga a buscar información para los equipos de salud a fin de tratar de manera mas integral el daño provocado por el accidente e indudablemente, ello tiene que ser tomado en consideración en la prevención y en los proyectos terapéuticos curativos de la lesión.

A todo lo anteriormente señalado hay que agregar que las reacciones de las personas ante una situación estresante, en éste caso una quemadura, recibe el nombre de "Estrategias de Afrontamiento". Lazarus y Folkman (1986) definen este concepto, como aquellos esfuerzos cognitivos y conductuales constantemente cambiantes que se desarrollan para manejar las demandas específicas externas y/o internas que son evaluadas como excedentes o desbordantes de la cultura del individuo. Estos mismos autores, proponen la siguiente taxonomía para clasificar el afrontamiento el cual consta de 8 dimensiones:

Confrontación: La cual describe acciones di- rectas orientadas a alterar la situación y sugiere un grado de hostilidad y toma de riesgo.

Distanciamiento: Se refiere a los esfuerzos cognitivos por separarse y minimizar el significado de la situación, por ejemplo, olvidar, negarse a tomar en serio la situación, comportarse como si nada hubiese ocurrido.

Autocontrol: Describe esfuerzos de regularse a sí mismo en cuanto a sus sentimientos y acciones, como por ejemplo, guardar los problemas para sí mismo y procurar no precipitarse.

Búsqueda de Apoyo Social: Describe los esfuerzos para buscar información de apoyo, soporte tangible y emocional en el entorno social.

Aceptación de la responsabilidad: La persona identifica su propio error en el problema y trata de corregir la situación (disculparse, criticarse a sí mismo).

Escape-evitación: Describe esfuerzos, deseos y pensamientos de escapar o evitar la situación (esperar que ocurra un milagro, tomar alcohol o drogas, etc).

Resolución Planificada de Problemas: Describe deliberados esfuerzos orientados al problema para alterar la situación. Se aprecia un enfoque analítico de resolver el problema (establecer un plan de acción y seguirlo).

Reevaluación Positiva: Describe esfuerzos de crear significados positivos de la situación al enfocarse en el desarrollo personal. Además, posee una dimensión religiosa que orienta a una interpretación positiva del problema.

Apreciamos entonces, que los padres poseen una variada gama de posibilidades de afrontamiento frente a un accidente de quemadura y además hemos señalado, la influencia que sus reacciones pueden tener sobre las conductas y las emociones de sus hijos. Entonces, es por todo lo expuesto que en esta investigación, nos hemos propuesto indagar sobre el fenómeno de las estrategias de afrontamiento 
utilizadas por los padres, frente a las secuelas de quemaduras visibles de su hijos, que se encuentran en los tres primeros años del período de rehabilitación en COANIQUEM. De esta manera, es que nos propusimos averiguar si existen relaciones entre las diversas formas de afrontamiento parental y los niveles de ansiedad manifestados por los niños quemados entre 6 y 9 años de edad.

Consideramos que este tema y en general el del tratamiento de la quemadura, ha sido poco estudiado y ha tenido escasa difusión en el área de la Psicología, prevaleciendo el tratamiento médico. No obstante, en la actualidad se reconoce que una quemadura no sólo deja secuelas a nivel físico sino también a nivel psicológico afectando además, al grupo familiar del niño quemado y a su entorno social. Es en este sentido, que consideramos que la relevancia de la presente investigación radicó en obtener información que pueda ser útil a los equipos interdisciplinarios que trabajan en los Centros de Salud donde se acoge al niño quemado y su familia; útil a los profesionales que se desempañan en los Centros de Rehabilitación de Quemados a fin de que puedan intervenir en los niños a través de sus padres y útil al trabajo del profesional psicólogo. En definitiva, creemos que es relevante el buscar información a fin de fomentar en los padres de niños quemados, estrategias relacionadas con bajos niveles de ansiedad, a objeto que los menores puedan reducir los costos emocionales de la quemadura. Por último, consideramos que la información que se obtuvo en ésta investigación, no sólo se puede utilizar respecto de lo curativo, sino también en acciones de promoción y educación respecto de las reacciones de los mayores cuando un menor sufre este tipo de accidente.

\section{Metodología}

El presente es un estudio que se enmarca dentro de la metodología cuantitativa. El diseño es de carácter no experimental, transversal y descriptivo-correlacional (Baptista,
Fernández \& Hernández, 1998; Sierra-Bravo, 1995).

El universo de este estudio corresponde a niños(as) entre 6 y 9 años que se encuentran en los tres primeros años de tratamiento en el Centro de Rehabilitación COANIQUEM y padres o tutores del niño que lo acompañan en el proceso de rehabilitación.

La muestra es no probabilística (Baptista, et al., 1998) y estuvo conformada por 49 niños(as) y sus respectivos padres y/o tutores (49) sumando un número total de 98 sujetos.

Los criterios de selección para la muestra infantil consideraron a niños (as) de entre 6 y 9 años, con secuela en una zona visible (cara, cuello y extremidades) y que se encontraran dentro de los primeros 36 meses de rehabilitación. En cuanto a los criterios de selección para la muestra parental se consideraron al padre, madre o tutor que se halla responsabilizado del cuidado del niño (a) durante su tratamiento en COANIQUEM.

\section{Instrumentos}

Para realizar este estudio se utilizaron dos instrumentos de evaluación: Uno de ellos fue el Cuestionario de Modos de Afrontamiento (Lazarus \& Folkman, 1986), el cual consta de 66 ítems que se evalúan en una escala tipo likert de 4 posiciones, donde la persona señala el grado de acuerdo a lo planteado en cada uno de los distintos ítems (Alamos, 1994 citado en Garfias, Godoy y Moncada, 2000).

Para esta aplicación, se utilizaron las modificaciones lingüísticas propuestas por Garfias, et. al. (2000) para facilitar la comprensión de los ítems en la realidad nacional dado que este test no ha sido estandarizado en Chile.

El segundo instrumento utilizado fue el Inventario de Ansiedad Estado/Rasgo para niños (STAIC), creado por Spielberger (1973) y adaptado a Chile por Cambriaso y Villaseca (1992) Se puede aplicar individual o colectiva- 
mente a niños de 9 a 15 años (o para niños menores que tengan una capacidad de lectura y comprensión suficiente) (Echeverría, 1996).

El inventario consta de 40 elementos, distribuidos en dos escalas. Una mide Ansiedad Estado y otra escala mide Ansiedad Rasgo, siendo los ítems de la primera escala diseñados para medir estados transitorios de Ansiedad. Por su parte, la escala Ansiedad Rasgo, está diseñada para medir diferencias individuales, relativamente estables en la predisposición a la ansiedad (Spielberger, 1973 citado en Cambriaso \& Villaseca, 1992).

ElSTAIC tiene un coeficiente de confiabilidad alto (alfa de Cronbach) de 0.82-0.87 para la escala Ansiedad Estado y 0.78 y 0.81 para la escala Ansiedad Rasgo. Así mismo posee un coeficiente de validez concurrente alto (0.75) con otras escalas de ansiedad como la Escala de Ansiedad Manifiesta Infantil (CMAS) y 0.63 con la General Anxiety Scale (GASC) (Spielberger, 1973 citado en Cambriaso \& Villaseca, 1992).

\section{Procedimiento}

Para llevar a cabo el estudio, se solicitó al Departamento de Informática de COANIQUEM un listado de pacientes (niños) que cumplieran los criterios de selección mencionados anteriormente. Este listado incluía las horas de controles médicos del año en curso lo cual permitió acceder a los niños seleccionados cuando asistían a control médico durante los meses de Enero y Febrero del 2003. Sin embargo, debido al elevado ausentismo a los controles médicos, se decidió implementar una nueva estrategia para contactar a la muestra, invitando telefónicamente a los padres y sus hijos a participar en la investigación de acuerdo a su disponibilidad. Una vez realizado el primer contacto personal con los padres/tutores y el niño(a), se les explicó el estudio y se solicitó su consentimiento por escrito para participar en la investigación junto con su hijo.
Se aplicaron ambos instrumentos en una única oportunidad y fueron administrados en duplas de investigadoras. El lugar de aplicación correspondió a distintas salas de atención según disponibilidad de espacio y tiempo de COANIQUEM. Además, se aplicó al padre/tutor una encuesta confeccionada por las autoras para obtener datos psicosociales de caracterización de la muestra.

Finalmente, se procedió a realizar una revisión de fichas para obtener datos tales como: extensión de la quemadura, grado de la quemadura, pronóstico, etc. Esta información fue necesaria conseguir, dado que no todos los padres/tutores manejaban los antecedentes médicos referentes a la quemadura de su hijo.

Con todos los datos obtenidos se procedió a realizar análisis descriptivos y correlacionales mediante el programa computacional SPSS versión 10.0.

\section{Resultados}

\section{Descriptivos y correlacionales}

La muestra infantil estuvo conformada por 49 niños correspondiendo el 55,1\% de los menores al sexo femenino y el $44,9 \%$ al sexo masculino.

Con respecto a las variables relacionadas con la quemadura se puede observar que la mayor parte de los niños $(73,5 \%)$ presentó una quemadura de tipo $\mathrm{AB}$ o de espesor intermedio; que el mayor número de niños $(59,2 \%)$ se encuentra en el primer año de tratamiento en COANIQUEM; y que casi el 80\% de los menores quedará con algún tipo de secuela (leve y / o profunda) como consecuencia del accidente.

La muestra parental, estuvo compuesta por 49 padres / tutores que acompañaron a los menores durante su proceso de tratamiento en COANIQUEM.

Con respecto a la descripción de las variables del estudio, se puede señalar que la variable Ansiedad Estado presenta una media de 29,5 puntos y una desviación típica de 5,3 puntos; y que la variable Ansiedad 
Rasgo presenta una media de 36 puntos y una desviación típica de 6,4 puntos.

En relación a las Estrategias de afrontamiento se puede observar que los padres manifestaron mayor uso de las estrategias Reinterpretación positiva ('x:18,1 ); Autocontrol ('x:14,0 ) y Resolución planificada ('x:13,5 ); siendo las menos utilizadas Aceptación de la responsabilidad (' $x: 8,9$ ); Confrontación (' $x: 8,8$ ) y Distanciamiento ('x:7,3 ).

Los resultados de la correlación entre las estrategias de afrontamiento de los padres y los niveles de ansiedad en los niños muestran que (ver Tabla 1):

La estrategia de afrontamiento Resolución planificada se correlaciona negativamente con los niveles de Ansiedad Rasgo (r: -0,422; $\mathrm{p}<0,01)$, lo cual indica que a mayor uso de esta estrategia de afrontamiento, menores los niveles de ansiedad rasgo en los niños (as).

La estrategia de afrontamiento Aceptación de la responsabilidad se correlaciona negati- vamente con los niveles de Ansiedad Estado ( $\mathrm{r}:-0,311 ; \mathrm{p}<0,05)$, lo cual indica que a mayor uso de esta estrategia, menores son los niveles de ansiedad estado en los niños(as).

La estrategia de afrontamiento Autocontrol se correlaciona negativamente con los niveles de Ansiedad Rasgo ( $\mathrm{r}:-0,288 ; \mathrm{p}<0,05)$, lo cual mostraría que a mayor utilización de esta estrategia por parte de los padres/tutores, los niños(as) mostrarían menores niveles de ansiedad rasgo.

La estrategia de afrontamiento Reinterpretación positiva se correlaciona negativamente con los niveles de Ansiedad Rasgo (r: $-0,323$; $\mathrm{p}<0,05)$; lo que indica que a mayor uso de esta estrategia, menores los niveles de ansiedad rasgo en los niños.

Las estrategias de afrontamiento búsqueda de apoyo social, distanciamiento, confrontación y escape-evitación, tuvieron cierto grado de correlación con la variable ansiedad, pero ésta no fue significativa.

Tabla 1. Correlaciones entre Estrategias de afrontamiento y Ansiedad E-R

\begin{tabular}{|l|l|r|r|}
\hline \multicolumn{2}{|c|}{} & Estado & Rasgo \\
\hline Confrontación & Correlación de Pearson &,- 167 &, 052 \\
& Sig. (bilateral) &, 252 &, 721 \\
\hline Distanciamiento & Correlación de Pearson &, 051 &,- 253 \\
& Sig. (bilateral) &, 730 &, 079 \\
\hline Autocontrol & Correlación de Pearson &,- 162 &,$- 288^{*}$ \\
& Sig. (bilateral) &, 267 &, 045 \\
\hline Búsqueda de apoyo & Correlación de Pearson &,- 123 &,- 267 \\
social & Sig. (bilateral) &, 401 &, 063 \\
\hline Aceptación de la & Correlación de Pearson &,$- 311^{*}$ &,- 070 \\
responsabilidad & Sig. (bilateral) &, 030 &, 631 \\
\hline Escape-evitación & Correlación de Pearson &, 191 &, 005 \\
& Sig. (bilateral) &, 189 &, 973 \\
\hline Resolución Planificada & Correlación de Pearson &,- 102 &,$- 422^{* *}$ \\
& Sig. (bilateral) &, 484 &, 003 \\
\hline Rinterpretación Positiva & Correlación de Pearson &,- 138 &,$- 323^{*}$ \\
& Sig. (bilateral) &, 346 &, 024 \\
\hline
\end{tabular}

* La correlación es significativa al nivel 0,05 (bilateral).

** La correlación es significativa al nivel 0,01 (bilateral). 
También se correlacionó la variable Ansiedad Estado-Rasgo con otras variables que están implicadas en el accidente de quemadura y que podrían influir en los mayores o menores niveles de ansiedad, como son la edad, la profundidad, el tiempo de tratamiento, el pronóstico y la extensión; encon- trándose que sólo la variable profundidad (variable recodificada) se correlacionó significativamente con la Ansiedad Rasgo. Dicha correlación fue positiva $(\mathrm{r}=0,33 ; \mathrm{p}<0,01)$, es decir, a mayor profundidad de la quemadura mayores los niveles de Ansiedad rasgo en los niños (ver tabla 2).

Tabla 2. Correlaciones entre Ansiedad E-R y otras variables relacionadas con las quemaduras

\begin{tabular}{|c|c|c|c|}
\hline & & A. Estado & A. Rasgo \\
\hline Edad & $\begin{array}{l}\text { Correlación de Pearson } \\
\text { Sig. (unilateral) }\end{array}$ & $\begin{array}{r}-, 190 \\
, 191\end{array}$ & $\begin{array}{l}\text {,038 } \\
, 798\end{array}$ \\
\hline Profundidad & $\begin{array}{l}\text { Correlación de Pearson } \\
\text { Sig. (unilateral) }\end{array}$ & $\begin{array}{r}-, 02 \\
, 859\end{array}$ & $\begin{array}{l}33^{*} \\
, 018\end{array}$ \\
\hline Tiempo de tratamiento & $\begin{array}{l}\text { Correlación de Pearson } \\
\text { Sig. (unilateral) }\end{array}$ & $\begin{array}{r}-, 13 \\
, 34\end{array}$ & $\begin{array}{r}-, 11 \\
, 43\end{array}$ \\
\hline Pronóstico & $\begin{array}{l}\text { Correlación de Pearson } \\
\text { Sig. (bilateral) }\end{array}$ & $\begin{array}{r}-, 001 \\
, 997\end{array}$ & $\begin{array}{l}128 \\
, 379\end{array}$ \\
\hline Extensión & $\begin{array}{l}\text { Correlación de Pearson } \\
\text { Sig. (bilateral) }\end{array}$ & $\begin{array}{r}-, 063 \\
, 669\end{array}$ & $\begin{array}{l}248 \\
, 086\end{array}$ \\
\hline
\end{tabular}

** La correlación es significativa al nivel 0,01 (unilateral).

\section{Discusión y Conclusiones}

Los resultados de este estudio muestran concordancia con lo propuesto por Lazarus y Folkman (1988, citado en Sandín, 1995) referente a las estrategias que se utilizan en el proceso de salud-enfermedad. Las estrategias de afrontamiento mayormente utilizadas por los padres son la Reinterpretación positiva, el Autocontrol y la Resolución planificada, que justamente corresponden a las tres estrategias que se correlacionan negativamente de forma significativa con los niveles de ansiedad rasgo. Respecto a las otras estrategias, se ha observado que dependen más del estresor mismo, de los recursos y limitaciones que la persona tenga para afrontar la situación.

También se pudo observar, que los niños en promedio presentaron niveles de ansiedad rasgo más altos que los niveles de ansie- dad estado. Este hecho, concuerda con lo planteado por Ewert y Yachán (1986) quienes señalan que en el niño el accidente de quemadura no sólo deja secuelas a nivel físico sino que también a nivel psicológico. Una de estas secuelas psicológicas es la ansiedad la que puede permanecer en el tiempo si es reforzada por el entorno social (como el colegio, estrategias de afrontamiento ineficaces del padre o falta de información, etc.), lo cual en vez de disminuir los niveles de ansiedad rasgo los mantendría o aumentaría, estableciéndose un patrón estable de respuesta frente a los estresores.

El tema de la percepción de control, también podría explicar el que los niveles de ansiedad estado se reduzcan en el menor, debido a factores como el aumento de la información que se le entrega al niño de acuerdo a su nivel de desarrollo, al apoyo emocional y al uso de estrategias eficaces por parte de los padres. Ello 
aumentaría la sensación de controlabilidad del niño, disminuyendo así la respuesta emocional de ansiedad estado. También se ha observado que con el tiempo el niño se va habituando a algunos aspectos de la quemadura y su tratamiento, lo cual posiblemente disminuye la ansiedad estado frente a estas variables contextuales ya experimentadas.

Con respecto a la contrastación de hipótesis, se puede señalar que el supuesto general de esta investigación el cual postula que se encontrarán diferencias en los niveles de ansiedad de los niños quemados según el tipo de estrategia de afrontamiento utilizada por sus padres se cumple, dado que algunas estrategias de afrontamiento de los padres se relacionan significativamente con menores niveles de ansiedad en los niños quemado.

Específicamente, con respecto a la primera hipótesis que señala que la Estrategia Confrontación se correlacionará con altos niveles de ansiedad en los niños, no se cumple debido a que no presenta una correlación significativa con el nivel de ansiedad en los niños. Esto último, se podría explicar dado que el padre al evaluar que puede hacer algo concreto para solucionar el problema (Lazarus \& Folkman, 1986), sentiría mayor control sobre la situación. Pero, al prevalecer en esta estrategia una conducta hostil e impulsiva más que una reflexión o una planificación acerca de lo que se puede hacer, entorpecería la posible ayuda que se le puede brindar a un hijo en estas circunstancias.

La segunda hipótesis que señala que la Estrategia de Afrontamiento Resolución Planificada de Problemas se correlacionará con bajos niveles de ansiedad en los niños, se cumplió parcialmente dado que la estrategia Resolución planificada se correlacionó negativamente con niveles de ansiedad rasgo, sin embargo, no se encontró una relación significativa entre esta estrategia y ansiedad estado. Lo anterior, podría ser explicado ya que si bien esta estrategia, al igual que la anterior se centra en la solución del problema, se diferencia de esta última debido a que los esfuerzos se centran en analizar una situación y planear los pasos a seguir, caracterizando a esta estrategia principalmente como cognitiva, reflexiva y ausente de toma de riesgo y hostilidad.

La tercera hipótesis señala que la Estrategia de Afrontamiento Distanciamiento se correlacionará con altos niveles de ansiedad en los niños, no se comprobó debido a que la estrategia distanciamiento no correlacionó significativamente con los niveles de ansiedad en los menores. Esta estrategia se centra en la emoción y conlleva una minimización del significado de la situación por parte del padre que podría, por un lado, aumentar la ansiedad en el niño (al no ser una fuente de apoyo) y por otro lado, podría disminuirla (al restarle importancia a la situación a los ojos del niño, éste podría creer que su problema no es tan importante) por lo tanto esta estrategia depende de otras variables, como por ejemplo, la independencia/dependencia del niño hacia sus padres, autoestima, presencia apoyo en el entorno familiar, principalmente.

La cuarta hipótesis que señala que la Estrategia de Afrontamiento Autocontrol, se correlacionará con bajos niveles de ansiedad en los niños se comprueba parcialmente dado que esta estrategia sólo se correlaciona significativamente con la ansiedad rasgo, no observándose correlación con la ansiedad estado. Esto se podría explicar dado que el padre al regular sus emociones y acciones no demuestra descontrol de éstas últimas, favoreciendo una actitud tranquila y calmada frente al acontecimiento independiente de las emociones que sienta por él. Este comportamiento organizado en los padres actúa como factor protector para el niño en su vivencia frente al accidente. Lo anterior, se puede relacionar con la hipótesis de "Crisis parental" postulada por Bush y cols. (1988, citado en Méndez \& Ortigosa, 2000) quienes plantean que los padres que presentan un comportamiento desorganizado en situaciones estresantes, tendrán hijos que desarrollarán me- 
canismos de ansiedad ante el estresor en vez de conductas adecuadas de afrontamiento, es decir, el padre al no manifestar desorganización en sus acciones contribuye a disminuir los niveles de ansiedad en el niño.

Así mismo, el hecho de que el padre se involucre en adquirir conocimientos respecto a la enfermedad de su hijo y logre establecer una adecuada perspectiva de ésta, contribuirá a que el menor maneje una visión realista de su enfermedad y logre una mejor adaptación a su tratamiento permitiéndole la expresión verbal de temores y dudas; de esta manera los padres logran contener emocionalmente a sus hijos lo cual repercute en un mejor manejo de la ansiedad.

La quinta hipótesis no se cumple debido a que la estrategia Aceptación de la responsabilidad no se correlaciona positivamente con los niveles de ansiedad, sin embargo, se correlaciona negativamente con ansiedad estado, o sea, que a mayor utilización de esta estrategia menores serán los niveles de ansiedad estado. Respecto a la ansiedad rasgo, no se encontró una correlación significativa entre ella y la estrategia de Aceptación de la responsabilidad.

Respecto a esta estrategia, se cree que el padre al identificar su propio error en el problema acepta su responsabilidad en el accidente de su hijo, pudiendo experimentar sentimientos de culpa. Lo anterior concuerda con lo propuesto por Bendlin, et. al. (1993) quienes postulan que una de las causas principales de estrés emocional para los padres y otros miembros de la familia es el sentimiento de culpabilidad, se hayan visto envueltos directamente en el accidente o no.

Los sentimientos de culpa de los padres se agravarían al observar el sufrimiento de su niño y la impotencia para mitigarlo, además del conocimiento de que quedarán cicatrices permanentes (Bendlin, et. al. 1993). El padre para mitigar estos sentimientos puede acercarse emocionalmente a su hijo favoreciendo una relación de apoyo con el niño, lo que podría explicar que en esta investigación se hallan encontrado datos que indican una correlación negativa con la ansiedad estado, sin embargo, si estos sentimientos de culpa llevan a los padres a consentir y sobreproteger en extremo a su niño se dificultará la adaptación de su hijo a la secuela de quemadura.

La sexta hipótesis no se cumple debido a que la estrategia Escape-evitación no se correlaciona significativamente con los niveles de ansiedad. Lo anterior confirma lo planteado por Lazarus y Folkman (1988), quienes plantean que este tipo de estrategia no tiene una asociación clara con los resultados relacionados al proceso de salud-enfermedad, dado que los autores postulan que esta estrategia dependería de factores contextuales y transaccionales. Desde este punto de vista, si consideramos que el accidente de quemadura es un acontecimiento de tipo irreversible donde no se puede cambiar la situación, el sujeto puede afrontar el problema evitando o escapando. Si esto ocurre, el padre no asumiría los sentimientos negativos que la situación le genera, debido a que se aleja del estresor para reducir la ansiedad, sin embargo, si la utilización de esta estrategia se rigidiza, no se podría realizar la elaboración de la experiencia vivida.

La séptima hipótesis se comprueba parcialmente, dado que la estrategia Reinterpretación positiva se correlaciona positivamente con los niveles de ansiedad rasgo, es decir, que a mayor utilización de esta estrategia menores serán los niveles de ansiedad rasgo en los niños. En cuanto a la ansiedad estado, no correlacionó significativamente con esta estrategia. Esta forma de afrontamiento crea significados positivos de una situación al enfocarse en el desarrollo personal y posee una dimensión religiosa que orienta a una interpretación positiva del problema. En la presente investigación el 98\% de la muestra se adscribía a alguna creencia religiosa lo que concuerda con la alta utilización de esta es- 
trategia. Si se toma en cuenta que la idiosincrasia chilena valida la fe y la creencia religiosa (Garfias et. al. 2000), es posible que esto halla orientado a los encuestados hacia esta tendencia.

Esta estrategia al cambiar el significado de una situación negativa a una visión positiva también mitiga los efectos de la situación estresante dado que busca obtener algo beneficioso de la experiencia, transmitiéndola así hacia el niño. Esta influencia parental, según Palomo del Blanco (1995), se debe a que en general en los niños resulta de capital importancia la valoración que del suceso hagan los padres; una respuesta positiva de éstos puede amortiguar el impacto estresante sobre el niño, por el contrario si el progenitor se muestra ansioso ante el acontecimiento el niño fácilmente imitará la respuesta emocional del adulto.

También se puede señalar que al otorgarle el padre una significación positiva a la experiencia estresante de la quemadura, se tiene una vivencia más esperanzadora con respecto al tratamiento y al pronóstico de la quemadura, aumentando la confianza y la seguridad en los resultados del tratamiento, por lo tanto disminuyendo la incertidumbre que tenga el padre.

La octava hipótesis no se comprueba, dado que la Búsqueda de apoyo social no se correlaciona significativamente con los niveles de ansiedad en los niños. Sin embargo en los resultados del estudio se observa una tendencia a correlacionar esta estrategia negativamente con los niveles de ansiedad rasgo, es decir que a mayor uso de búsqueda de apoyo social menores serán los niveles de ansiedad. Esto se apoya en lo postulado por Lazarus y Folkman (1986) quienes señalan que la búsqueda de apoyo social actúa como factor protector para prevenir experiencias dañinas o amenazantes así como para proporcionar recursos valiosos para el manejo de la situación, dando información, contención emocional, y apoyo instrumental. Sin embargo, los mismos autores postulan que esta estrategia suele estar influida por factores contextuales y transaccionales lo que hace que no haya una asociación clara entre esta estrategia y el proceso de salud enfermedad de las personas.

Por otro lado, también se correlacionó la variable ansiedad con otras variables para ver si éstas tenían algún grado de relación con la ansiedad estado-rasgo. En primer lugar, se analizó la relación entre ansiedad y edad del grupo de niños no encontrándose una correlación significativa entre ambas, sin embargo se puede observar una leve asociación negativa, es decir, que a mayor edad de los niños menores niveles de ansiedad estado. Lo anterior se apoya en los planteamientos de Méndez y Ortigosa (2000), quienes señalan que el conocimiento y la experiencia a la que tiene acceso el niño mayor contribuye a mitigar la ansiedad.

En segundo lugar, se estudió la relación entre ansiedad y profundidad de la quemadura, encontrándose una correlación significativa (positiva) entre profundidad y ansiedad rasgo, es decir, que a mayor profundidad de la quemadura mayores niveles de ansiedad rasgo. Esto se puede explicar dado que una quemadura de mayor profundidad dejaría secuelas físicas más severas, lo que influiría en el autoconcepto y en la forma que el niño se relacione con los demás, pudiendo presentar temor a la reacción que los otros tengan de él, miedo al rechazo y a las múltiples agresiones del medio particular y general lo que según Ewertz y Yachán (1984) favorecería la aparición de mayores niveles de ansiedad.

Otra explicación que podría influir en la relación ansiedad/profundidad es la que hace referencia al tema del dolor. Según Ramírez (2001), el dolor experimentado por los pacientes quemados favorece la aparición de ansiedad por lo que se podría decir que las quemaduras que producen mayor dolor (quemaduras tipo AB o intermedias) aumentarían los niveles de ansiedad en el niño. 
En tercer lugar, se correlacionó la variable ansiedad con el tiempo de tratamiento que lleva el niño en COANIQUEM, no encontrándose correlaciones significativas entre estas variables. Sin embargo, se puede observar una ligera disminución de la ansiedad a medida que transcurre el tiempo de tratamiento. La literatura informa que los primeros dos años de tratamiento son los más difíciles, dado que comienza el período de resocialización en el cual aparecen las dificultades de interacción social, la tendencia al aislamiento y la confrontación del niño de su nueva imagen física con el entorno social (Castillo y Santander, 1996). Pero a medida que el niño comienza a reasumir sus actividades normales puede adaptarse a los cambios ocurridos y tiene mayor probabilidad de recuperar las funciones previas al accidente de quemadura.

En cuarto lugar, se correlacionó la variable ansiedad con pronóstico de la secuela de quemadura no encontrándose una correlación significativa entre ambas variables. Es importante señalar, que según lo observado en las entrevistas, las expectativas de los padres respecto al pronóstico de sus hijos eran positivas por lo cual éste más que una fuente de ansiedad fue una fuente esperanzadora lo cual coincide con una de las estrategias más utilizadas como es la reinterpretación positiva y que a su vez se correlaciona con bajos niveles de ansiedad.

Finalmente, se correlacionó la variable ansiedad con extensión de la quemadura no encontrándose tampoco una correlación significativa entre ambas. No obstante, se puede apreciar una tendencia a correlacionar positivamente, o sea, que a mayor extensión de la quemadura mayores serían los niveles de ansiedad rasgo, lo que puede deberse a que frente a un incremento de la extensión aumentaría el impacto de la quemadura para el niño y su entorno social. En este sentido, diversos autores proponen que sufrir cualquier quemadura, excepto las más insignificantes, es un acontecimiento traumático en la vida de un niño debido a que una quema- dura puede convertirlo en un ser dependiente (Artz \& Moncrief, 1972).

Si bien esta investigación tomó un solo polo de la relación padre-hijo (se midieron los niveles de ansiedad a la luz de las estrategias utilizadas por el padre) no quiere decir que esta relación sea unidireccional. En este sentido, García (2002) postula que es común pensar en la influencia de los padres sobre la psicología del hijo, pero se subestiman los efectos que produce éste sobre sus progenitores. De acuerdo a esto, las influencias se pueden presentar en ambos sentidos, lo cual podría ser el objetivo de una futura investigación.

\section{Referencias}

Artz, C \& Moncrief, J. (1972). Tratado de quemaduras. México: Nueva Editorial Interamericana.

Baptista, P., Fernández, C.\& Hernández, R. (1998). Metodología de la investigación. (2 ${ }^{\mathrm{a}}$ Ed.). México: Ed. Mc Graw-Hill

Bendlin, L., Linares, M. \& Benaim, D. (1993). Tratado de quemaduras. Cap. 43: Rehabilitación psicosocial y vocacional. México: Ed. Interamericana-Mc Graw Hill

Buendía, J. \& Mira, J. (1993). Eventos vitales, afrontamiento y desarrollo: un estudio sobre el estrés infantil. Murcia: Universidad de Murcia.

Cambriaso, C.\& Villaseca M., (1992). Adaptación y estandarización del Inventario de Ansiedad Estado-Rasgo, para niños de 9 a 12 años (STAIC) de la provincia de Santiago. Tesis para optar al título de Licenciado en Psicología, Universidad Diego portales, Santiago-Chile

Capdeville, M.\& Medina, B. (1998). Diseño, aplicación y evaluación de un programa de intervención psicológica dirigido a padres de niños quemados. Tesis para optar al grado de licenciado en psicología. Universidad Central, Santiago de Chile. 
Castillo, C.\& Santander, D. (1996). Propuesta de un modelo de atención psicológica al niño quemado. Revista Terapia Psicológica, No26, Año XIV, Vol. VI (2).

Echeverría, E. (1996). Trastornos de Ansiedad en la infancia. Madrid: Ed. Pirámides.

Ewertz, M., \& Yachán, M. (1984). Aspectos Psicosociales del niño quemado. Revista Terapia Psicológica. Vol. 3, No 4, pág. 91-94.

García, J., (2002). La familia en el desarrollo psicológico del niño. Revista Mexicana de Puericultura y Pediatría, $N^{\circ}$ 55, Vol. 10, pp. 26-36.

Garfias, X., Godoy, J. \& Mondaca, H. (2000). Estudio comparativo de estrategias de afrontamiento entre mujeres con cáncer de mama y mujeres sanas. Tesis para optar al título de Psicólogo. Universidad de Santiago de Chile.

Hermosilla, W., Montt, M. (2001). Trastorno de estrés post-traumático en niños. $R e$ vista Chilena de NeuroPsiquiatría, Vol. 39, pp. 110-120.

Lazarus, R.\& Folkman, S. (1986). Estrés y procesos cognitivos. Barcelona: Ed. Martínez Roca.
Méndez, F. \& Ortigosa, J. (2000). Hospitalización infantil: Repercusiones psicológicas, teoría y práctica. Madrid: Ed. Biblioteca Nueva.

Palanca, I \& Pedreira, J. (2002). Psiconcología Pediátrica. Extraído el 29 de marzo, 2003 de <www.psiquiatria.com/psiquiatria/ revista/79/7351/?++interactivo $>$

Palomo del Blanco, M. (1995). El niño hospitalizado, características, evaluación y tratamiento. Madrid: Ed. Pirámide

Ramírez, J. (2001). Las reacciones emocionales del paciente quemado. Extraído el 10 de mayo, 2002, de <http://pp.terra.com.mx L inaq/doc1.html>

Sandín, B. (1995). Manual de Psicopatología. Madrid: Ed. Mc Graw-Hill.

Sierra-Bravo, R. (1995). Técnicas de Investigación Social: Teoría y Ejercicios. Madrid: Ed. Paraninfo.

Ugalde, I. (1998). Accidentes por quemaduras en niños. Extraído el 10 de mayo , 2002 de <www.unam.mx/rompan/49/rf49 rep.html> 